\title{
Magnetoelastic couplings in the distorted diamond-chain compound azurite
}

\author{
Pham Thanh Cong, ${ }^{1}$ Bernd Wolf,,${ }^{*}$ Rudra Sekhar Manna, ${ }^{1}$ Ulrich Tutsch, ${ }^{1}$ Mariano de Souza, ${ }^{1,2}$ \\ Andreas Brühl, ${ }^{1}$ and Michael Lang ${ }^{1}$ \\ ${ }^{1}$ Physics Institute, Goethe University Frankfurt(M), D-60438 Frankfurt(M), Germany \\ ${ }^{2}$ Instituto de Geociências e Ciências Exatas - IGCE, Unesp - Universidade Estadual Paulista, Departamento de Física, Caixa Postal 178, \\ 13506-970 Rio Claro (SP), Brazil
}

(Received 30 January 2014; revised manuscript received 24 April 2014; published 27 May 2014)

\begin{abstract}
We present results of ultrasonic measurements on a single crystal of the distorted diamond-chain compound azurite $\mathrm{Cu}_{3}\left(\mathrm{CO}_{3}\right)_{2}(\mathrm{OH})_{2}$. Pronounced elastic anomalies are observed in the temperature dependence of the longitudinal elastic mode $c_{22}$ which can be assigned to the relevant magnetic interactions in the system and their couplings to the lattice degrees of freedom. From a semiquantitative analysis of the magnetic contribution to $c_{22}$ the magnetoelastic coupling $G=\partial J_{2} / \partial \epsilon_{b}$ can be estimated, where $J_{2}$ is the intradimer coupling constant and $\epsilon_{b}$ the strain along the intrachain $b$ axis. We find an exceptionally large coupling constant of $|G| \sim 3650 \mathrm{~K}$ highlighting an extraordinarily strong sensitivity of $J_{2}$ against changes of the $b$-axis lattice parameter. These results are complemented by measurements of the hydrostatic pressure dependence of $J_{2}$ by means of thermal expansion and magnetic susceptibility measurements performed both at ambient and finite hydrostatic pressure. We propose that a structural peculiarity of this compound, in which $\mathrm{Cu}_{2} \mathrm{O}_{6}$ dimer units are incorporated in an unusually stretched manner, is responsible for the anomalously large magnetoelastic coupling.
\end{abstract}

DOI: 10.1103/PhysRevB.89.174427

PACS number(s): 75.45. $+\mathrm{j}, 72.55 .+\mathrm{s}, 75.80 .+\mathrm{q}$

\section{INTRODUCTION}

Low-dimensional quantum-spin systems have attracted continuous attention due to the wealth of unusual magnetic properties that result from the interplay of low dimensionalty, competing interactions, and strong quantum fluctuations. Among these systems, the diamond chain has been of particular interest, where triangular arrangements of spin $S=1 / 2$ entities with exchange coupling constants $J_{1}, J_{2}$, and $J_{3}$ are connected to form chains [1-4]. In recent years, great interest has surrounded the discovery of azurite, $\mathrm{Cu}_{3}\left(\mathrm{CO}_{3}\right)_{2}(\mathrm{OH})_{2}$ [5], as a model system of a $\mathrm{Cu}^{2+}(S=1 / 2)$-based distorted diamond chain with $J_{1} \neq J_{2} \neq J_{3}$ [9]. The observation of a plateau at $1 / 3$ of the saturation magnetization [9] is consistent with a description of azurite in terms of an alternating dimer-monomer model [2,10]. Two characteristic temperatures (energies) have been derived from peaks in the magnetic susceptibility $\chi(T)[9,10]$. Whereas the peak at $T_{1}^{\chi} \simeq 25 \mathrm{~K}$ has been assigned to the dominant intradimer coupling $J_{2}$, the one at $T_{2}^{\chi} \simeq 5 \mathrm{~K}$ has been linked to a monomer-monomer coupling along the chain $b$ axis [9]. There have been conflicting results, however, as for the appropriate microscopic description of the relevant magnetic couplings of azurite [11-15]. Very recently, Jeschke et al. [16] succeeded in deriving an effective microscopic model capable of providing a consistent picture of most available experimental data for not too low temperatures, i.e., distinctly above the transition into long-range antiferromagnetic order at $T_{N}=1.86 \mathrm{~K}$ [17]. According to this work, the exchange couplings $J_{1}, J_{2}$, and $J_{3}$ are all antiferromagnetic, thus placing azurite in the highly frustrated parameter regime of the diamond chain. Within the "refined model" proposed there, $J_{2} / k_{B}=33 \mathrm{~K}$ and an effective monomer-monomer coupling $J_{m} / k_{B}=4.6 \mathrm{~K}$ were found.

\footnotetext{
*wolf@physik.uni-frankfurt.de
}

Another intriguing property of azurite, not accounted for so far from theory, refers to the strong magnetoelastic couplings in this compound. These couplings manifest themselves, e.g., in a pronounced structural distortion accompanying the magnetic transition at $T_{N}$, as revealed by thermal expansion $[18,19]$ and neutron scattering experiments $[19,20]$. Here we present a study of these magnetoelastic couplings of azurite by means of temperature-dependent measurements of the elastic constant and uniaxial thermal expansion coefficients. These data are supplemented by thermal expansion and susceptibility measurements under hydrostatic pressure conditions. The salient result of our study is the observation of an extraordinarily large magnetoelastic coupling constant of the intradimer coupling $J_{2}$ with respect to intrachain deformations. This coupling manifests itself in pronounced anomalies in the elastic constant and uniaxial thermal expansion coefficients; the latter are characterized by a negative Poisson effect. We propose that the anomalous magnetoelastic behavior of azurite is a consequence of the material's structural peculiarities, in particular, the presence of unusually stretched $\mathrm{Cu}_{2} \mathrm{O}_{6}$ dimer units.

\section{EXPERIMENTAL DETAILS}

The single crystals (samples 1-4) used for the measurements described in this paper were cut from a large high-quality single crystal which was also studied by neutron scattering and muon spin resonance ( $\mu \mathrm{SR})[12,19]$. For the ultrasonic experiments two parallel surfaces normal to the [010] direction were prepared and two piezoelectric polymer-foil transducers were glued to these surfaces. To determine the acoustic $c_{22}$ mode, longitudinal sound waves in the frequency range around 50-105 MHz were propagated along the [010] direction. In the following we will focus on the results obtained at $75 \mathrm{MHz}$ which exhibit the highest signal-to-noise ratio. By using a phase-sensitive detection technique [21] the relative change of the sound velocity and the sound attenuation were 
simultaneously measured as the function of temperature for $0.08 \mathrm{~K} \leqslant T \leqslant 310 \mathrm{~K}$. A top-loading dilution refrigerator was used for measurements at $T \leqslant 1.5 \mathrm{~K}$, whereas a ${ }^{4} \mathrm{He}$ bath cryostat, equipped with a variable temperature insert, was employed for accessing temperatures $T \geqslant 1.5 \mathrm{~K}$. The elastic constant $c_{i j}$ is obtained from the sound velocity $v_{i j}$ by $c_{i j}=$ $\rho v_{i j}^{2}$, where $\rho$ is the mass density. For measurements of the uniaxial thermal expansion coefficients, $\alpha_{i}(T)=l_{i}^{-1}\left(\partial l_{i} / \partial T\right)$, where $l_{i}(T)$ is the sample length along the $i$ axis, two different dilatometers were used. Experiments under ambient pressure along the $a^{\prime}, b$, and $c^{*}$ axes, where $a^{\prime}$ and $c^{*}$ are perpendicular to the (102) and (102) crystallographic planes, respectively, were carried out by means of an ultrahigh-resolution capacitive dilatometer, built after Ref. [22], with a resolution of $\Delta l / l \geqslant$ $10^{-10}$. In addition, measurements along the $b$ axis were performed by using a different dilatometer [23], with a slightly reduced sensitivity of $\Delta l / l \geqslant 5 \times 10^{-10}$, enabling measurements to be performed under helium-gas pressure. The magnetic susceptibility at ambient pressure and at various finite pressure values was measured with a SQUID magnetometer (Quantum Design MPMS). For the measurements under pressure, a CuBe piston cylinder clamped cell was used with Daphne oil 7373 as a pressure-transmitting medium. At low temperature, the pressure inside the pressure cell was determined by measuring the superconducting transition temperature of a small piece of indium.

\section{RESULTS AND DISCUSSION}

\section{A. Elastic anomalies and pressure/strain dependence of the relevant magnetic energy scales}

Figure 1(a) shows the experimental results (open symbols) of the longitudinal elastic constant $c_{22}(T)$ of azurite (sample 1) over the whole temperature range investigated. For the frequency range 50-105 $\mathrm{MHz}$ investigated, no frequency dependence of the elastic constant $c_{22}(T)$ was observed. A similar behavior was reported earlier for the $c_{22}$ mode at temperatures around $T_{N}$ [25]. Upon cooling, the $c_{22}$ mode initially increases (hardening) as expected for materials where anharmonic phonon interactions dominate. Slightly below room temperature, however, a pronounced softening becomes visible which is accompanied by various anomalies at lower temperatures $T \leqslant 30 \mathrm{~K}$. These anomalies can be discerned particularly clearly in the inset of Fig. 1(a), where the lowtemperature data are shown on a logarithmic temperature scale. Most prominent is a distinct minimum at a temperature around $27 \mathrm{~K}$. Moreover, the data disclose a small dip slightly below $10 \mathrm{~K}$. The position of this feature is difficult to estimate due to its smallness and the strong variation of $c_{22}$ with temperature caused by the nearby anomalies. We assign these features [labeled $T_{1}^{c_{22}}$ and $T_{2}^{c_{22}}$ in the inset to Fig. 1(a)] to the characteristic temperatures $T_{1}$ and $T_{2}$ of azurite, as revealed by susceptibility measurements $[9,10]$. In addition, the elastic data highlight a sharp minimum around $1.9 \mathrm{~K}$, reflecting the transition into long-range antiferromagnetic ordering at $T_{N}=1.88 \mathrm{~K}$, and a steplike softening of comparable size to that at $T_{N}$ around $T_{0}=0.37 \mathrm{~K}$. The latter feature is likely to be of magnetic origin as well [24]. In contrast to the distinct softening observed in the $c_{22}(T)$ elastic constant,

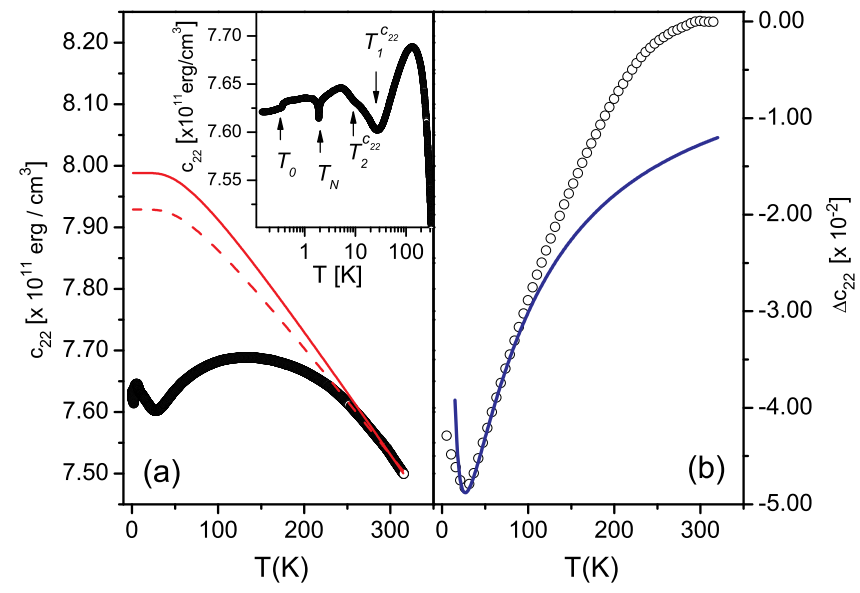

FIG. 1. (Color online) (a) Temperature dependence of the longitudinal elastic $c_{22}(T)$ mode (open circles) of single crystalline azurite (sample 1). The red solid (broken) line represents the nonmagnetic elastic background $c_{b g}(T)$ derived from fitting Eq. (1) to the experimental data for $T \geqslant 290 \mathrm{~K}(T \geqslant 250 \mathrm{~K})$; see text for details. Inset to (a): The longitudinal mode $c_{22}(T)$ on a logarithmic temperature scale. The arrows mark the positions of the elastic anomalies in $c_{22}$ which are connected to the various energy scales $\left(T_{1}^{c_{22}}, T_{2}^{c_{22}}\right)$, the phase transition into long-range antiferromagnetic order $\left(T_{N}\right)$, and a transition of unknown, most likely magnetic origin $\left(T_{0}\right)$. (b) Normalized magnetic contribution to the elastic $c_{22}$ mode $\Delta c_{22}(T)=\left[c_{22}(T)-c_{b g}(T)\right] / c_{b g}(T=0)$ as a function of temperature. The solid blue line represents a fit to the experimental data (open symbols) based on Eq. (2) for $15 \mathrm{~K} \leqslant T \leqslant 290 \mathrm{~K}$.

we note that no accompanying feature is revealed in the ultrasonic attenuation (not shown) for temperatures above $20 \mathrm{~K}$. However, pronounced anomalies in the attenuation were observed at $T_{N}$ [25] and around $10 \mathrm{~K}$, the latter corresponding to the energy scale of the Heisenberg chain not discussed here. This anomaly is similar to the one observed in the quasi-one-dimensional compound $\mathrm{CsNiCl}_{3}$ [26].

For a quantitative analysis of the magnetic contribution to $c_{22}$, the nonmagnetic (normal) elastic background $c_{b g}$ has to be subtracted from the data. By lacking a nonmagnetic reference material, isostructural to azurite, from which $c_{b g}$ could be determined experimentally, we used the phenomenological expression [27] for $c_{b g}(T)$

$$
c_{b g}(T)=c_{b g}^{0}-\frac{s}{e^{\frac{t}{T}}-1} .
$$

Here $c_{b g}^{0}$ is the value of the elastic constant at $T=0$, and $s$ and $t$ are constants. The quantity $t$ is usually set to $\Theta_{D} / 2$, where $\Theta_{D}$ is the Debye temperature (see Ref. [27] for details). By choosing $t=175 \mathrm{~K}$, corresponding to $\Theta_{D}=350 \mathrm{~K}$ as derived from specific heat [28], and by using $c_{b g}^{0}$ and $s$ as free parameters, Eq. (1) was fitted to the data for temperatures $290 \mathrm{~K} \leqslant T \leqslant 315 \mathrm{~K}$, i.e., high enough so that magnetic contributions can be safely neglected. The application of Eq. (1), which requires a three-dimensional, Debye-like phonon spectrum, is justified by specific heat measurements on azurite [28]. There, a consistent description of the total specific heat was obtained by using a Debye model for the lattice contribution. The elastic background 
obtained by this fitting procedure is displayed as the solid red line in Fig. 1(a). We note that due to the pronounced softening, with an onset slightly below room temperature, and the upper limit of our experiment of $315 \mathrm{~K}$, the fitting range for Eq. (1) is strongly limited which implies some uncertainty. For instance, by extending the lower bound of the fitting range to $250 \mathrm{~K}$, where softening is already visible, $c_{b g}^{0}$ is reduced by approximately $0.6 \%$ [broken line in Fig. 1(a)]. Figure 1(b) shows the normalized magnetic contribution to the elastic $c_{22}$ mode $\Delta c_{22}(T)=\left[c_{22}(T)-c_{b g}(T)\right] / c_{b g}(T=0)$ as a function of temperature (open symbols) obtained by subtracting the elastic background $c_{b g}(T)$ from the experimental data. Note that we refrain from correcting the experimental data for the thermal expansion because the relative length change between $200 \mathrm{~K}$ and $1.6 \mathrm{~K}$ amounts to only $1.9 \times 10^{-3}$. This is more than an order of magnitude smaller than the elastic softening and a factor of about 3 smaller than the uncertainty implied in the fitting procedure of the elastic background. The soderived magnetic contribution $\Delta c_{22}$ reveals a huge softening of approximately five percent on cooling down to $T_{1}^{c_{22}}$. The large softening indicates an extraordinarily strong magnetoelastic coupling which is likely of exchange-striction type for the following reason. For longitudinal modes, such as the $c_{22}$ mode investigated here, a two-ion magnetoelastic coupling arises from the modulation of the distance or bond angles between the magnetic ions which changes the interaction. Furthermore the single-ion magnetoelastic coupling for $\mathrm{Cu}^{2+}$ is small because of the vanishing quadrupole matrix elements [29]. In order to semiquantitatively evaluate the corresponding magnetoelastic coupling constant, we introduce in Eq. (2) a generalized strain susceptibility $\chi_{\text {str }}$. This model accounts, within a random-phase approximation (RPA) [29], for the temperature dependence of the elastic constants of coupled dimers characterized by an intradimer coupling constant corresponding to the dimers' singlet-triplet excitation gap $\Delta$. This results in a temperature dependence for the elastic constant [29] of

$$
c_{22}(T)=\frac{\partial^{2} F}{\partial\left(\varepsilon_{22}\right)^{2}}=c_{b g}(T)-N G^{2} \chi_{\text {str }}(T),
$$

where $F$ is the free energy, $\varepsilon_{22}$ is the strain along the [010] direction, and $G=\partial \Delta / \partial \varepsilon_{b}$ is the variation of the singlettriplet energy gap $\Delta$ of the dimers upon applying a $b$-axis strain; i.e., $G=\partial J_{2} / \partial \varepsilon_{b}$ for the case of azurite. $N$ is the density of dimers and

$$
\chi_{\mathrm{str}}(T)=\frac{\chi_{s}(T)}{1-K \chi_{s}(T)}
$$

is the generalized strain susceptibility. Here $\chi_{s}(T)=3 e^{-\Delta / k_{B} T} /\left(k_{B} T Z^{2}\right)$ denotes the strain susceptibility of a single dimer with $Z=1+3 e^{-\Delta / k_{B} T}$ the partition function and $K$ the strength of the effective magnetoelastic dimer-dimer interaction. Whereas Eq. (2) provides a very good description for systems with weakly dispersing triplet excitations, see, e.g., Ref. [30] for $\mathrm{SrCu}_{2}\left(\mathrm{BO}_{3}\right)_{2}$, where the excitations are localized [31], it may serve only as a rough approximation for azurite given its more complex excitation spectrum. According to inelastic neutron scattering (INS) experiments [12], singlet-triplet excitations of the dimers exist in azurite within a range of energies from $3.5 \mathrm{meV}$ up to about $7 \mathrm{meV}$, with high scattering intensities around $4.5 \mathrm{meV}$ and $5.8 \mathrm{meV}$. By lacking a model which adequately accounts for the strain susceptibility for such a band of excitations, we focus here only on an approximate description of the low-energy sector of azurite by modeling the spectrum with a single excited state (with a high density of states) separated from the singlet state by an energy gap of $4.5 \mathrm{meV}$, corresponding to $\Delta / k_{B}=52 \mathrm{~K}$. The latter value was also seen in ESR experiments [32] and was associated with the dominant magnetic coupling constant $J_{2}$. Accordingly, in our fitting procedure, we fixed the size of the gap to $\Delta / k_{B}=52 \mathrm{~K}$, consistent with these INS [12] and ESR [32] data.

The solid blue line in Fig. 1(b) shows a fit to the experimental data using Eq. (2) with a dimer density $N=$ $0.9918 \times 10^{22} \mathrm{~cm}^{-3}$, corresponding to 2 dimers per unit cell, and $c_{22}^{0}(T=0)=7.988 \times 10^{11} \mathrm{erg} / \mathrm{cm}^{3}$; the latter is taken from the fitting procedure of the elastic background. From a nonlinear least-squares fit, constrained to the temperature range $15 \mathrm{~K} \leqslant T \leqslant 290 \mathrm{~K}$, the parameters $K$ and $|G|$ can then be determined. The so-derived curve provides a good overall description of the data up to about $100 \mathrm{~K}$. In particular it describes well the minimum in $\Delta c_{22}(T)$. Since the position of the minimum in the model curve is dictated by $\Delta$, this agreement supports the above assumption that the minimum in $\Delta c_{22}(T)$ reflects singlet-triplet excitations related to $J_{2}$. The deviations of the fit from the data become significant at temperatures $T \leqslant 22 \mathrm{~K}$ [not shown in Fig. 1(b)]. This is attributed to the simultaneous action of additional magnetic couplings at lower temperatures, especially those related to $T_{2}$. On the other hand, the progressive deviations of the model curve from the data with increasing temperature above $100 \mathrm{~K}$ are attributed to the simplified energy spectrum considered in our model, where triplet excitations at higher energies $(E>\Delta)$ are neglected. Irrespective of these shortcomings, the fit allows for an order-of magnitude estimate of the magnetoelastic coupling constant $|G|$ to about $3650 \mathrm{~K}$. Although this number involves significant uncertainties, there is no doubt that it has to be of exceptionally large size to account for the observed softening in $c_{22}$ of approximately $5 \%$. This effect not only exceeds corresponding anomalies typically revealed for low-dimensional quantum spin systems $[33,34]$ by one to two orders of magnitude; it even exceeds the huge effects found for the longitudinal mode in the coupled-dimer system $\mathrm{SrCu}_{2}\left(\mathrm{BO}_{3}\right)_{2}$ [35]. The large $|G|$ value reflects an unusually high sensitivity of the dominant energy scale in azurite against the application of uniaxial strain. For the parameter $K$ in Eq. (3) the fit yields a negative value of about $-340 \mathrm{~K}$, indicating that the effective magnetoelastic dimer-dimer interaction is antiferrodistortive in azurite. This means that neighboring dimer units are shifted or rotated in opposite directions, as opposed to a ferrodistortive-type of interaction $(K>0)$, where the motions would happen in the same direction.

In order to obtain supplementary information about the magnetoelastic couplings corresponding to the various energy scales, we performed measurements of the coefficient of thermal expansion and the magnetic susceptibility both at ambient- and under hydrostatic-pressure conditions.

Figure 2(a) shows the results of the temperature dependence of thermal expansion coefficients $\alpha_{i}(T)\left(i=a^{\prime}, b, c^{*}\right)$ for temperatures $1.6 \mathrm{~K} \leqslant T \leqslant 75 \mathrm{~K}$ measured at ambient pressure. 


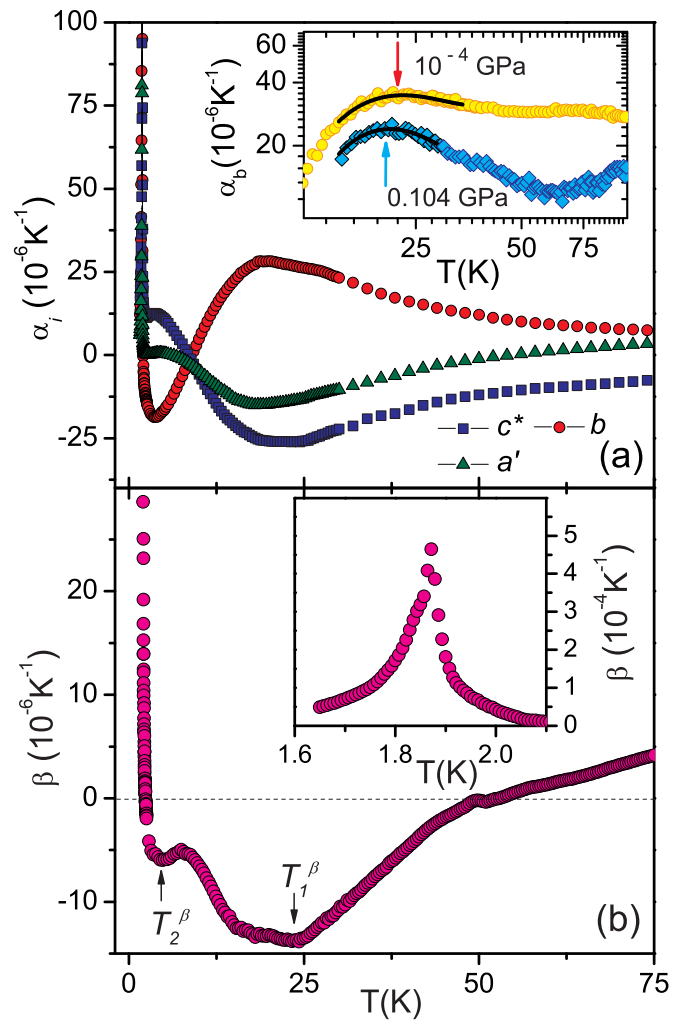

FIG. 2. (Color online) (a) Uniaxial coefficients of thermal expansion of single crystalline azurite (sample 3 ) along the chain $b$ axis and two orthogonal axes perpendicular to $b$ labeled $a^{\prime}, c^{*}$. Inset: Temperature dependence of the $b$-axis expansivity measured on crystal 4 for $20 \mathrm{~K} \leqslant T \leqslant 100 \mathrm{~K}$ at ambient pressure $\left(10^{-4} \mathrm{GPa}\right)$ (yellow circles) and at a helium-gas pressure of $0.104 \mathrm{GPa}$ (blue diamonds) in a double-logarithmic representation; cf. Ref. [23]. The arrows mark the position of the local maximum in $\alpha_{b}$ at $T_{1}$ derived from the fourth-order polynomial (black solid line) fitted to the experimental data. (b) Volume expansivity $\beta=\alpha_{a^{\prime}}+\alpha_{b}+\alpha_{c^{*}}$ determined from the data in (a). Arrows labeled $T_{1}^{\beta}$ and $T_{2}^{\beta}$ mark the position of anomalies (minima) in $\beta$. Inset: Details of the phase transition anomaly in $\beta$ at $T_{N}$.

Similar to the elastic constant, three distinct anomalies are observed in this temperature range along all three crystallographic axes. Upon cooling, the $b$-axis data show a broad positive maximum around $20 \mathrm{~K}$ whereas along the two other axes $\left(a^{\prime}\right.$ and $\left.c^{*}\right)$ a negative minimum appears. Upon further cooling to lower temperatures, a minimum shows up in the $b$-axis data, which contrasts with maxima around $3.5 \mathrm{~K}$ in the data along the $a^{\prime}$ and $c^{*}$ axes. Despite having opposite signs, the anomalies in $\alpha_{b}(T)$ and those in $\alpha_{a^{\prime}}(T)$ and $\alpha_{c^{*}}(T)$ do not cancel each other out in the volume expansion coefficient $\beta(T)=\alpha_{a^{\prime}}(T)+\alpha_{b}(T)+\alpha_{c^{*}}(T)$, shown in the main panel of Fig. 2(b). The volume expansivity exhibits a pronounced negative contribution, giving rise to a change of sign around $50 \mathrm{~K}$, and a broad minimum around $25 \mathrm{~K}$ followed by a second minimum at $\simeq 5 \mathrm{~K}$. The strong upturn in $\beta(T)$ and $\alpha_{i}$ at lower temperatures is due to the antiferromagnetic phase transition at $T_{N}$. This is shown more clearly in the inset of Fig. 2(b) where $\beta(T)$ is displayed on expanded scales around $T_{N}$. An extraordinarily large $\lambda$-type anomaly, lacking any hysteresis upon cooling and warming, is observed which demonstrates the second-order character of the phase transition at $T_{N}$. From the coincidence of the anomalies in $\beta(T)$ [and $\alpha_{i}(T)$ ] with those revealed in the magnetic susceptibility [9] and elastic constant (Fig. 1), we conclude that these anomalies reflect the characteristic temperatures $T_{1}$ and $T_{2}$ which are related to the energy scales $J_{2}$ and $J_{m}$, respectively. Thus from the evolution of these anomalies under pressure we may determine the pressure dependencies of these energy scales. In the inset of Fig. 2(a) we compare, on a logarithmic temperature scale, the temperature dependence of $\alpha_{b}$ for a small pressure of $10^{-4} \mathrm{GPa}$ with that of $0.104 \mathrm{GPa}$. These data, which have been taken on sample 4 by employing a different dilatometer, especially designed for measurements under helium-gas pressure [23], disclose two remarkable features. First, we find a strong change in the temperature dependence of $\alpha_{b}(T)$, accompanied by a considerable suppression in its absolute value over the whole temperature range investigated, i.e., for $T \leqslant 100 \mathrm{~K}$. Note that due to the solidification of the pressure medium, the measurements at $0.104 \mathrm{GPa}$ were limited to $T>14.5 \mathrm{~K}$. Since changes of the lattice expansivity under a pressure of $0.104 \mathrm{GPa}$ for a material with a normal bulk modulus are expected to be less than $1 \%$, see Ref. [23] and the discussion therein, we attribute these effects to the influence of pressure on the material's magnetic properties and their coupling to the lattice degrees of freedom. The presence of significant magnetic contributions at elevated temperatures $T \gg J_{2} / k_{B}$ which strongly couple to the lattice is consistent with the acoustic behavior revealed for the $c_{22}(T)$ elastic mode, yielding an onset temperature for the pronounced softening as high as $290 \mathrm{~K}$; cf. Fig. 1(a). Second, a thorough inspection of the data around the maximum reveals a shift of the position of the maximum to lower temperatures on increasing the pressure from $p=0.1$ to $0.104 \mathrm{GPa}$. This can be quantified more precisely by fitting both data sets in the immediate surrounding of the maximum by fourth-order polynomials [23], depicted as solid lines in Fig. 2(a). By identifying the position of the maximum with $T_{1}$, we find a pressure dependence $\partial T_{1} / \partial p=-(0.08 \pm 0.03) \mathrm{K} / \mathrm{GPa}$.

Figure 3 displays the data of the magnetic susceptibility $\chi(T, p=$ const. $)$ as a function of temperature for $2 \mathrm{~K} \leqslant T \leqslant$ $300 \mathrm{~K}$ at varying pressure values from ambient pressure up to $0.62 \mathrm{GPa}$. The ambient-pressure data are consistent with those reported by Kikuchi et al. [9], yielding an increase in $\chi$ with decreasing temperature and two broadened maxima at $T_{1}^{\chi}$ and $T_{2}^{\chi}$. Upon increasing the pressure we observe a progressive increase of the low-temperature susceptibility; cf. Fig. 3. In addition, a closer look at the low-temperature data in the inset of Fig. 3 discloses a shift of the position of both maxima to lower temperatures albeit at different rates.

For a quantitative analysis of the susceptibility data for not too low temperatures around $T_{1}$ and up to $300 \mathrm{~K}$, we again use an RPA-molecular field expression for coupled dimers, in analogy to the procedure applied for analyzing the elastic constant $c_{22}(T)$ data:

$$
\chi_{m}(T)=\frac{\chi_{0}(T)}{1-\widetilde{K} \chi_{0}(T)}
$$




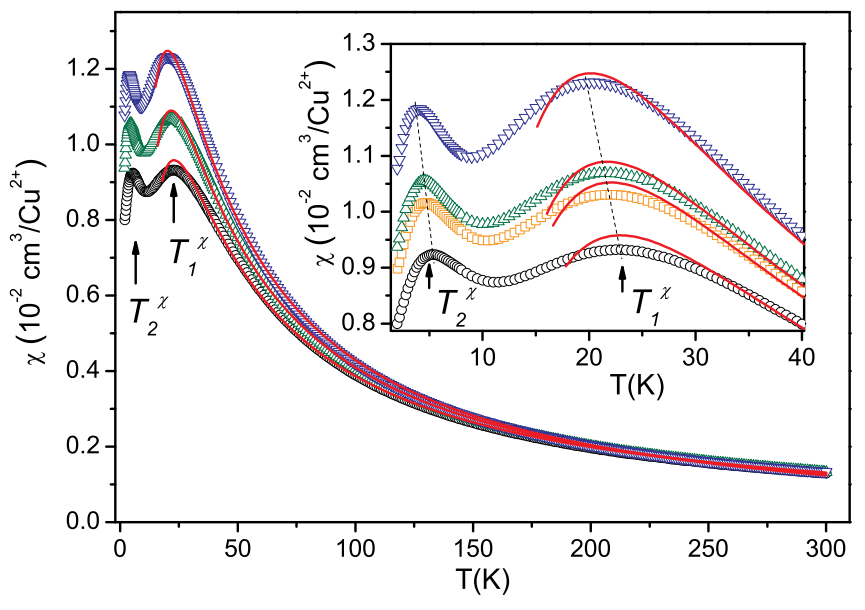

FIG. 3. (Color online) Temperature dependence of magnetic susceptibility of single crystalline azurite (sample 2) measured at various hydrostatic pressure values (from bottom to top ambient pressure, 0.36, $0.62 \mathrm{GPa}$ ) in a magnetic field of $B=2 \mathrm{~T}$ applied parallel to the $b$ axis. Red solid lines correspond to fits to the data based on a model for interacting dimers given in Eq. (4). Inset: Blow-up of the data (symbols) and model curves (red lines) in the vicinity of the characteristic temperature $T_{1}^{\chi}$. Besides the data at ambient pressure (black circles), $p=0.360 \mathrm{GPa}$ (green triangles) and $0.62 \mathrm{GPa}$ (purple triangles), also data at $0.26 \mathrm{GPa}$ (orange squares) are shown. Arrows labeled $T_{1}^{\chi}$ and $T_{2}^{\chi}$ mark the position of the maxima in the various data sets. Broken lines indicate the shift in the maximum position with pressure.

Here $\chi_{0}(T)=2 e^{-\Delta / k_{B} T} /\left(k_{B} T Z\right)$ is the magnetic susceptibility of an isolated dimer, $Z$ the partition function, and $\widetilde{K}$ an average magnetic interdimer coupling. A fit to the experimental data at ambient pressure was performed for $15 \mathrm{~K} \leqslant T \leqslant 300 \mathrm{~K}$ by using Eq. (4) and a Curie contribution, according to the amount of Cu-monomer spins, with $\Delta=J_{2}$ and $\widetilde{K}$ as free parameters. This fit provides a very good description of the data including the height and the position of the maximum at $T_{1}^{\chi}$; cf. the solid red line running through the ambient-pressure data points in Fig. 3. From the fit we obtain $J_{2} / k_{B}=(40.5 \pm 0.7) \mathrm{K}$, consistent with the value suggested by DFT calculations for the "full model" discussed there [16], and $\widetilde{K}=(4.1 \pm 0.8) \mathrm{K}$. Note that this value of $\widetilde{K}$ indicates that the average magnetic dimer-dimer interaction is small and ferromagnetic. The RPAmolecular field description remains good also for the data taken at finite pressure. For these fits the same Curie susceptibility as used for the ambient-pressure data was used to account for the magnetic contributions of the monomers. The evolution of the parameters $J_{2}$ and $\widetilde{K}$ with pressure, derived from fitting Eq. (4) to the finite-pressure data, is shown in Fig. 4. We find a suppression of $J_{2}$ with increasing pressure which can be approximated by $\partial J_{2} / \partial p \approx-(0.077 \pm 0.007) \mathrm{K} / \mathrm{GPa}$. For the average magnetic interdimer interaction we find an approximate linear increase under pressure with a larger rate of $\partial \widetilde{K} / \underset{\sim}{\partial} p \approx(0.216 \pm 0.002) \mathrm{K} / \mathrm{GPa}$. This pronounced increase of $\partial \widetilde{K} / \partial p$ is responsible for the growth of the low-temperature susceptibility with pressure.

As will be discussed in more detail below (Sec. IIIC), the dominant ferromagnetic character of the dimer-dimer interaction can be assigned to a particular $\mathrm{Cu}-\mathrm{O}-\mathrm{Cu}$ exchange

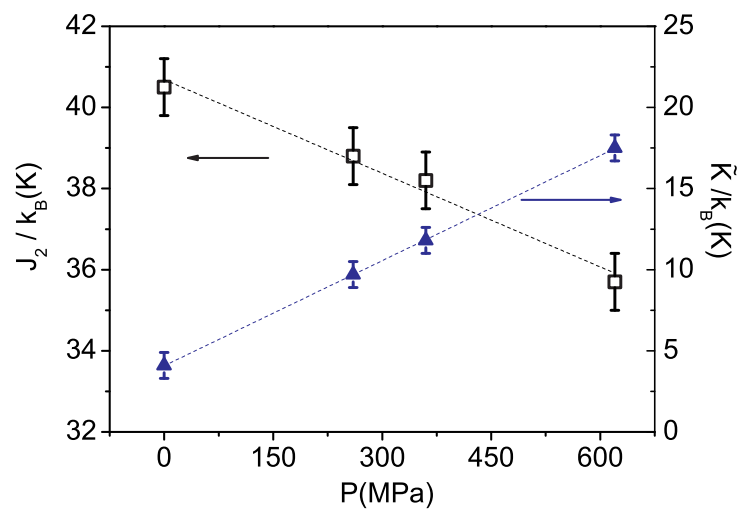

FIG. 4. (Color online) Evolution of the fit parameters, the intradimer coupling constant $J_{2}$ (open squares, left scale) and the average magnetic dimer-dimer interaction energy $\widetilde{K}$ (full triangles, right scale), with pressure as derived from fits of Eq. (2) to the data in Fig. 3.

path connecting dimers of adjacent chains along the $a$ direction.

\section{B. Magnetoelastic coupling at $\boldsymbol{T}_{N}$}

As shown in the previous section, the phase transition into the long-range antiferromagnetic order in azurite at $T_{N}=1.88 \mathrm{~K}$ is accompanied by a pronounced anomaly in the elastic $c_{22}$ mode, corresponding to a softening of about $0.1 \%$ [cf. inset to Fig. 1(b)] which exceeds by far the features usually revealed at a magnetic transition in low-dimensional spin systems where often only a kink-like anomaly is observed; see Refs. [34,36], for typical examples, and Ref. [29] for a review. This goes along with an extraordinarily large anomaly in the uniaxial coefficients of thermal expansion $\alpha_{i}$; see Fig. 2(b). As a result of the strong magnetoelastic coupling, there is a large $\lambda$-type anomaly in the volume expansion coefficient $\Delta \beta$ [see the inset of Fig. 2(b)] corresponding to a relative reduction of the volume upon cooling from 2 to $1.6 \mathrm{~K}$ of $\Delta V / V=-5.7 \times 10^{-5}$ as shown in Fig. 5 .

According to the Ehrenfest relation, the discontinuities at this second-order phase transition in $\beta, \Delta \beta$, and that in the specific heat, $\Delta C_{p}$, can be used to determine the pressure dependence of the Néel temperature in the limit of vanishing pressure

$$
\left(\frac{\partial T_{N}}{\partial p}\right)_{p \rightarrow 0}=V_{\mathrm{mol}} T_{N} \frac{\Delta \beta}{\Delta C_{p}} .
$$

By using $\Delta \beta=(550 \pm 30) \times 10^{-6} \mathrm{~K}^{-1}$ and $\Delta C_{p}=(6.18$ $\pm 0.4) \times \mathrm{J} \mathrm{mol}^{-1} \mathrm{~K}^{-1}$ taken from Ref. [12], we find $\left(\frac{\partial T_{N}}{\partial p}\right)_{p \rightarrow 0}=(0.15 \pm 0.02) \mathrm{K} / \mathrm{GPa}$. This extraordinarily large pressure dependence, exceeded only by some exceptional cases, such as the coupled antiferromagnetic/structural transition in Co-substituted $\mathrm{CaFe}_{2} \mathrm{As}_{2}$ [38], highlights the strong magnetoelastic coupling and the unusual elastic properties of azurite.

The large $\Delta \beta$ at $T_{N}$, tantamount to a large pressure dependence of $T_{N}$ in Eq. (5), is partly due to the fact that in azurite at $T_{N}$ the discontinuities in the uniaxial expansion coefficients, 


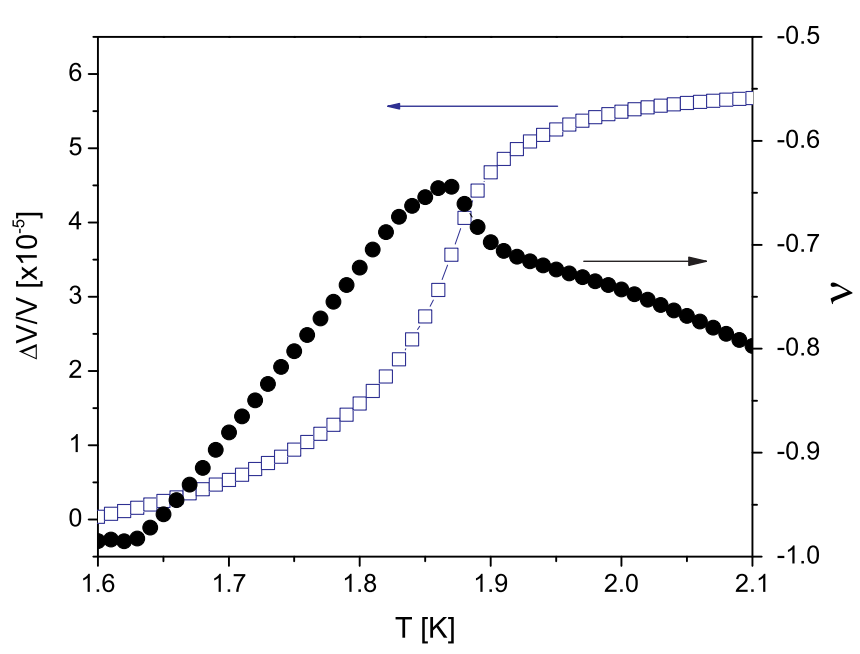

FIG. 5. (Color online) Poisson ratio (black full circles, right scale) together with the relative volume change around $T_{N}$ (blue open squares, left scale).

$\Delta \alpha_{i}$, all have the same (positive) sign. Hence, this phase transition is characterized by an anomalous Poisson effect.

In general for an isotropic material, the Poisson ratio, which measures the material's cross section under tension, is defined as

$$
v=-\frac{\epsilon_{y}}{\epsilon_{x}},
$$

where $\epsilon_{x}$ is the strain in stretching direction and $\epsilon_{y}$ perpendicular to it. In most materials $v$ is positive, which reflects the fact that an expansion along one axis is usually accompanied by a compression in the perpendicular direction, to keep the overall volume change small. In many materials $v$ values are found in the range $0.2 \leq 0.5$ [39]. The latter value corresponds to the situation that the material keeps its volume under tension. A positive $v<0.5$ means that the material becomes thinner when it is stretched, the behavior encountered for most materials. In contrast, materials with a negative Poisson ratio become thicker when they are stretched. Those compounds, called auxetic materials, are of interest due to potential technical applications [39].

In Fig. 5 we show the Poisson ratio $v$ of azurite for temperatures around $T_{N}$. For the present anisotropic case, $v$ has been determined by using the relative length changes $(\Delta l / l)_{b}$ [corresponding to the integral of $\alpha_{b}(T)$ with respect to temperature] for the strain $\epsilon_{x}$ along the stretching $b$ direction and $\overline{\epsilon_{y}}=\left(\epsilon_{a^{\prime}}+\epsilon_{c^{*}}\right) / 2=\left[(\Delta l / l)_{a^{\prime}}+(\Delta l / l)_{c^{*}}\right] / 2$ perpendicular to it. We stress that $v$ of azurite reaches a normal value of 0.23 around room temperature (not shown). However, as indicated in Fig. 5, $v$ exhibits a large negative value of -0.8 for temperatures slightly above $T_{N}$. This value increases to -0.65 upon cooling to $T_{N}$ below which it further decreases, reaching almost -1 at $1.6 \mathrm{~K}$. Note that this value is close to the stability limit of any elastic linear, isotropic material where the requirement of positive Young's shear and bulk moduli dictates $v>-1$.

\section{Relationship between structural and magnetic properties}

We start the discussion by considering the dimer-dimer interaction $\widetilde{K}$ revealed from the analysis of the susceptibility measurements under variable pressure. The dominant ferromagnetic character of this interaction is assigned to the $\mathrm{Cu} 2-\mathrm{O} 3-\mathrm{Cu} 2$ exchange path [cf. Fig. (6a)] connecting dimers of adjacent chains along the $a$ axis. The corresponding $\mathrm{Cu} 2-\mathrm{O} 3-\mathrm{Cu} 2$ bond angle amounts to $91.57^{\circ}$ at $5 \mathrm{~K}$, consistent with a weak ferromagnetic interaction as revealed for hydroxobridged $\mathrm{Cu}$ (II) complexes [37]. Note that also the DFT calculations for the "full model" of azurite exhibit a small ferromagnetic exchange [16]. It is likely that under hydrostatic pressure the structure will deform in a way such that this angle decreases with increasing pressure. This is consistent with an increase of the ferromagnetic interdimer coupling $\widetilde{K}$ derived from susceptibility measurements under pressure; cf. Fig. 4.

In the following we will argue that the auxetic behavior at $T_{N}$ and the huge magnetoelastic coupling of azurite is likely due to peculiarities of the molecular arrangement in this compound, in particular that of the $\mathrm{Cu}_{2} \mathrm{O}_{6}$ dimer units; cf. Fig. 6. As shown in Fig. 6(b), $\mathrm{CuO}_{4}$ monomer units (containing $\mathrm{Cu} 1$ ) are connected via $\mathrm{O} 2$ ions to $\mathrm{Cu}_{2} \mathrm{O}_{6}$ dimers

(a)
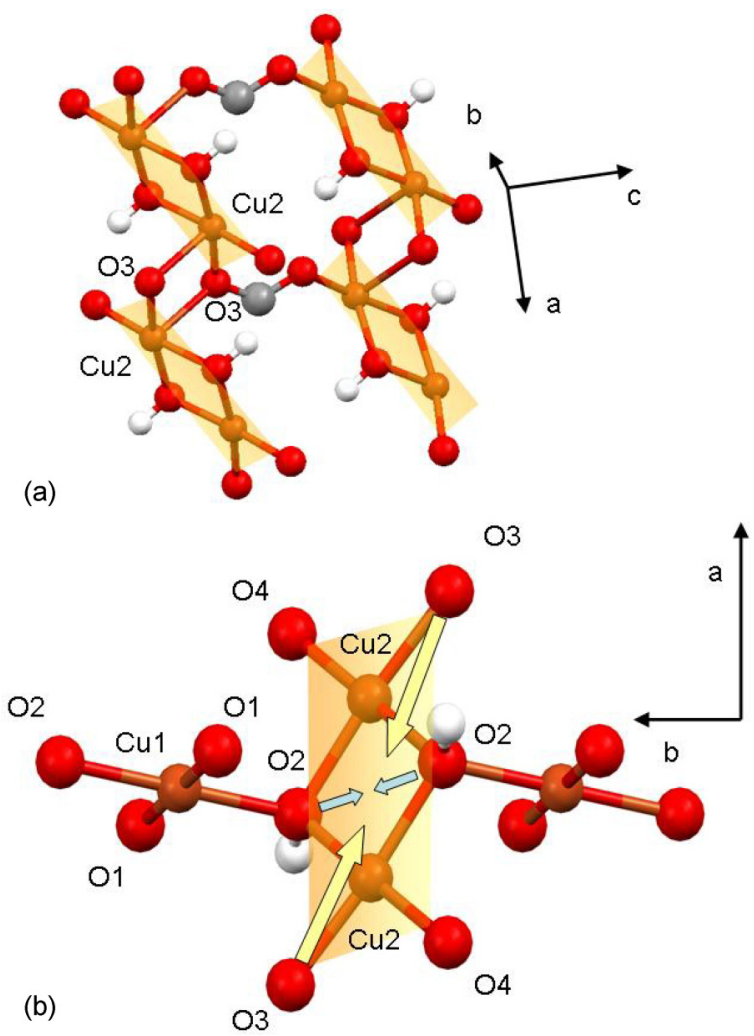

FIG. 6. (Color online) Sections of the structure of azurite. (a) The dominant interdimer interaction, connecting dimers (marked by yellow planes) in adjacent chains along the $a$ direction, is mediated via the $\mathrm{Cu} 2-\mathrm{O} 3-\mathrm{Cu} 2$ exchange path. (b) Arrangement of relevant structural units of azurite including two $\mathrm{Cu}(\mathrm{II})$ monomers $\left(\mathrm{CuO}_{4}\right.$ containing $\mathrm{Cu} 1)$ and one dimer $\left(\mathrm{Cu}_{2} \mathrm{O}_{6}\right.$ containing $\left.\mathrm{Cu} 2\right)$ forming chains along the $b$ axis viewed perpendicular to the $a b$ plane. The labels correspond to the room temperature structure reported in Ref. [20]. 
TABLE I. Structural data of the $\mathrm{Cu}_{2} \mathrm{O}_{6}$ dimer units of azurite at $300 \mathrm{~K}$ and $5 \mathrm{~K}$ taken from Refs. [8,20]. The first column denotes the atoms involved with labels according to Fig. 6. The second (third) column gives the distance $d$ between these atoms at $300 \mathrm{~K}(5 \mathrm{~K})$. The fourth column gives the difference in these distances upon cooling from $300 \mathrm{~K}$ to $5 \mathrm{~K}$. The $\mathrm{O} 2-\mathrm{O} 2$ and $\mathrm{O} 3-\mathrm{O} 3$ distances, showing the strongest changes upon cooling and which are involved in the auxetic behavior, are printed in bold.

\begin{tabular}{lccr}
\hline \hline & $\begin{array}{c}\text { distance }(\AA) \\
\text { at } 300 \mathrm{~K}\left(d_{300 K}\right)\end{array}$ & $\begin{array}{c}\text { distance }(\AA) \\
\text { at } 5 \mathrm{~K}\left(d_{5 K}\right)\end{array}$ & $d_{300 K}-d_{5 K}(\AA)$ \\
\hline $\mathrm{O} 3-\mathrm{Cu} 2$ & 1.93850 & 1.93320 & 0.00530 \\
$\mathrm{Cu} 2-\mathrm{O} 4$ & 1.93990 & 1.93910 & 0.00080 \\
$\mathrm{O} 2-\mathrm{Cu} 2$ & 1.99470 & 1.98820 & 0.00650 \\
$\mathrm{Cu} 2-\mathrm{O} 2$ & 1.96750 & 1.96420 & 0.00330 \\
$\mathrm{Cu} 2-\mathrm{Cu} 2$ & 2.98510 & 2.99220 & -0.00710 \\
$\mathbf{O 2 - O 2}$ & $\mathbf{2 . 6 0 5 6 0}$ & $\mathbf{2 . 5 8 3 2 0}$ & $\mathbf{0 . 0 2 2 4 0}$ \\
$\mathbf{O 3}-\mathrm{O3}$ & $\mathbf{6 . 2 0 1 8 0}$ & $\mathbf{6 . 1 9 1 3 0}$ & $\mathbf{0 . 0 1 0 5 0}$ \\
\hline
\end{tabular}

(containing $\mathrm{Cu} 2$ and marked by yellow planes in Fig. 6) to form chains along the $b$ axis. According to structural data at room temperature [8] and $5 \mathrm{~K}$ [20], cf. Table I, the structural parameters of the monomers, involving O1-Cu1 and $\mathrm{O} 2-\mathrm{Cu} 1$ bonds, change very little upon cooling and are very close to those values (1.90-1.93 $\AA$ ) typically found in isolated $\mathrm{Cu}$ (II) complexes [37]. This indicates a stable and rigid configuration of the $\mathrm{CuO}_{4}$ monomer units. In contrast, the structural parameters of the $\mathrm{Cu}_{2} \mathrm{O}_{6}$ dimers are rather unusual. In particular the $\mathrm{Cu} 2-\mathrm{O} 2$ bonds, mediating the intradimer coupling $J_{2}$, are significantly longer (1.9947 $\AA$ and $1.9675 \AA$ at $300 \mathrm{~K}$ ) (see Table I) than those found in isolated dimer complexes, reflecting an unusually "stretched" arrangement. It is thus obvious to suspect that this dimer unit represents the flexible part in the structure which may accommodate itself accordingly when the surrounding structure is exposed to external stimuli. This is the case, e.g., when a $b$-axis strain is applied or, alternatively, upon cooling through $T_{1}$ which is accompanied by a significant contraction of the $b$ axis (large positive anomaly in $\alpha_{b}$; cf. Fig. 2). In fact, according to structural data (Table I), it is the $\mathrm{O} 2-\mathrm{O} 2$ distance, as indicated by the blue arrows in Fig. 6(b), across the dimer which yields the strongest reduction upon cooling from $300 \mathrm{~K}$ to $5 \mathrm{~K}$, reflecting the softness of these dimer units. Note that changes of the $\mathrm{Cu} 2-\mathrm{O} 2$ geometry imply changes of the dominant magnetic interaction $J_{2}$. As Fig. 6(b) suggests, such a contraction along the $b$ direction will cause not only a reduction of the $\mathrm{O} 2-\mathrm{O} 2$ distance. It will also lead to changes of the $\mathrm{Cu} 2-\mathrm{O} 2$ bond length and the $\mathrm{Cu} 2-\mathrm{O} 2-\mathrm{Cu} 2$ bond angle $\left(97.77^{\circ}\right.$ at $\left.300 \mathrm{~K}\right)$ within the dimers. In fact, the $5 \mathrm{~K}$ structure reveals a $\mathrm{Cu} 2-\mathrm{O} 2-\mathrm{Cu} 2$ angle of $98.41(7)^{\circ}$. At the same time it is conceivable that upon the reorientation of the dimer in response to a $b$-axis strain, the tensile forces acting on the dimers get weaker, allowing the dimers to adopt a more natural, compact configuration, consistent with the observed reduction of the $\mathrm{Cu} 2-\mathrm{O} 2$ bond lengths. This less-strained dimer configuration is also visible in the intradimer O3-O3 distance which is reduced as well [see Table I and the yellow arrows in Fig. 6(b)]. Due to the orientation of the dimer unit in the $a c$ plane, a reduction of the $b$ axis is connected with a reduction of the $a$ and $c$ axes which then explains the anomalous Poisson effect [40] at low temperatures.

As for the anomalously large magnetoelastic coupling $|G|$, one might expect that the stretched arrangement of the dimers may alter the relationship between the exchange coupling constant and the dimers' structural parameters $\eta$, such as the interatomic distances or bonding angles, and with it the generalized derivatives $\partial J / \partial \eta$. According to Crawford et al. [37], who investigated various stable hydroxo-bridged dimer complexes, there is a linear correlation between the intradimer coupling and both the $\mathrm{Cu}-\mathrm{Cu}$ distance as well as the $\mathrm{Cu}-\mathrm{O}-\mathrm{Cu}$ bond angle. All of these materials exhibit nearly the same typical $\mathrm{Cu}-\mathrm{O}$ distances. It is likely that these relations may change significantly for a strongly stretched configuration, as realized for the dimer units in azurite. This may result in strongly enhanced derivatives $\partial J / \partial \eta$ such as the large magnetoelastic coupling constant $\partial J / \partial \epsilon_{b}$ revealed here. A microscopic theory, addressing the relationship between the coupling constants and the dimers' structural parameters, is necessary to confirm this conjecture. Note that the presence of prestrained structural units in azurite is consistent with the fact that a hydrothermal synthesis technique operating at a pressure of about $0.350 \mathrm{GPa}$ has to be applied for growing single crystals of this mineral [41].

\section{CONCLUSIONS}

Measurements of the longitudinal elastic constant $c_{22}$ and the uniaxial thermal expansion coefficients $\alpha_{i}$ on single crystalline azurite reveal pronounced anomalies associated with the intradimer coupling constant $J_{2}$. From a semiquantitative analysis of the elastic constant data, an exceptionally large value of the magnetoelastic coupling $G=\partial J_{2} / \partial \epsilon_{b}$ of $|G| \sim$ $3650 \mathrm{~K}$ has been derived. By lacking a microscopic theory, we tentatively assign this large value, exceeding corresponding magnetoelastic couplings for other low-dimensional quantum spin systems by two to three orders of magnitude, to structural peculiarities of azurite. We propose that it is the $\mathrm{Cu}_{2} \mathrm{O}_{6}$ dimer unit, which is incorporated in the structure in an unnaturally stretched manner, which is responsible for the exceptionally large magnetoelastic coupling in this system.

\section{ACKNOWLEDGMENTS}

We acknowledge financial support by the Deutsche Forschungsgemeinschaft via the SFB/TR49, and B. Lüthi, R. Valentí, and H. Jeschke for useful discussions.
[1] K. Takano, K. Kubo, and H. Sakamoto, J. Phys.: Condens. Matter 8, 6405 (1996).

[2] K. Okamoto, T. Tonegawa, and M. Kaburagi, J. Phys.: Condens. Matter 15, 5979 (2003).
[3] T. Tonegawa, K. Okamoto, T. Hikihara, Y. Takahashi, and M. Kaburagi, J. Phys. Soc. Jpn. Suppl. A 69, 332 (2000).

[4] T. Tonegawa, K. Okamoto, T. Hikihara, Y. Takahashi, and M. Kaburagi, J. Phys. Chem. Solids 62, 125 (2001). 
[5] Azurite crystallizes in the monoclinic space group $P 2_{1} / c$, first discovered by Gattow and Zeeman [6] and later confirmed by two independent investigations $[7,8]$.

[6] G. Gattow and J. Zemann, Acta Cryst. 11, 866 (1958).

[7] F. Zigan and H. D. Schuster, Z. Kristallogr. 135, 416 (1972).

[8] E. L. Belokoneva, Yu. K. Gubina, and J. B. Forsyth, Phys. Chem. Miner. 28, 498 (2001).

[9] H. Kikuchi, Y. Fujii, M. Chiba, S. Mitsudo, T. Idehara, T. Tonegawa, K. Okamoto, T. Sakai, T. Kuwai, and H. Ohta, Phys. Rev. Lett. 94, 227201 (2005).

[10] E. Frikkee and J. Handel, Physica 28, 269 (1962).

[11] H. J. Mikeska and C. Luckmann, Phys. Rev. B 77, 054405 (2008).

[12] K. C. Rule, A. U. B. Wolter, S. Süllow, D. A. Tennant, A. Brühl, S. Köhler, B. Wolf, M. Lang, and J. Schreuer, Phys. Rev. Lett. 100, 117202 (2008).

[13] B. Gu and G. Su, Phys. Rev. Lett. 97, 089701 (2006).

[14] Y. C. Li, J. Appl. Phys. 102, 113907 (2007).

[15] J. Kang, C. Lee, R. K. Kremer, and M-H. Whangbo, J. Phys.: Condens. Matter 21, 392201 (2009).

[16] H. Jeschke, I. Opahle, H. Kandpal, R. Valenti, H. Das, T. Saha-Dasgupta, O. Janson, H. Rosner, A. Brühl, B. Wolf, M. Lang, J. Richter, S. Hu, X. Wang, R. Peters, T. Pruschke, and A. Honecker, Phys. Rev. Lett. 106, 217201 (2011).

[17] R. D. Spence and R. D. Ewing, Phys. Rev. 112, 1544 (1958).

[18] F. Wolff-Fabris, S. Francoual, V. Zapf, M. Jaime, B. Scott, S. Tozer, S. Hannahs, T. Murphy, and A. Lacerda, J. Phys.: Conf. Ser. 150, 042030 (2009).

[19] M. C. R. Gibson, K. C. Rule, A. U. B. Wolter, J.-U. Hoffmann, O. Prokhnenko, D. A. Tennant, S. Gerischer, M. Kraken, F. J. Litterst, S. Süllow, J. Schreuer, H. Luetkens, A. Brühl, B. Wolf, and M. Lang, Phys. Rev. B 81, 140406(R) (2010).

[20] K. C. Rule, M. Reehuis, M. C. R. Gibson, B. Ouladdiaf, M. J. Gutmann, J.-U. Hoffmann, S. Gerischer, D. A. Tennant, S. Süllow, and M. Lang, Phys. Rev. B 83, 104401 (2011).

[21] B. Lüthi, G. Bruls, P. Thalmeier, B. Wolf, D. Finsterbusch, and I. Kouroudis, J. Low Temp. Phys. 95, 257 (1994).

[22] R. Pott and R. Schefzyk, J. Phys. E 16, 444 (1983).

[23] R. S. Manna, B. Wolf, M. de Souza, and M. Lang, Rev. Sci. Instrum. 83, 085111 (2012).

[24] P. T. Cong, B. Wolf, U. Tutsch, K. Removic-Langer, J. Schreuer, S. Süllow, and M. Lang, J. Phys.: Conf. Ser. 200, 012226 (2010).
[25] P. T. Cong, B. Wolf, R. S. Manna, A. Brühl, S. Köhler, and M. Lang, J. Korean Phys. Soc. 62, 2193 (2013).

[26] Y. Trudeau, M. Poirier, and A. Caillé, Phys. Rev. B 46, 169 (1992).

[27] Y. P. Varshni, Phys. Rev. B 2, 3952 (1970).

[28] H. Kikuchi, Y. Fujii, M. Chiba, S. Mitsudo, T. Idehara, T. Tonegawa, K. Okamoto, T. Sakai, T. Kuwai, K. Kindo, A. Matsuo, W. Higemoto, K. Nishiyama, M. Horvatic, and C. Bertheir, Prog. Theor. Phys. Suppl. 159, 1 (2005).

[29] B. Lüthi, Physical Acoustics in the Solid State (Springer, Berlin, 2005).

[30] S. Zherlitsyn, S. Schmidt, B. Wolf, H. Schwenk, B. Lüthi, H. Kageyama, K. Onizuka, Y. Ueda, and K. Ueda, Phys. Rev. B 62, R6097 (2000).

[31] H. Kageyama, M. Nishi, N. Aso, K. Onizuka, T. Yosihama, K. Nukui, K. Kodama, K. Kakurai, and Y. Ueda, Phys. Rev. Lett. 84, 5876 (2000).

[32] H. Ohta, S. Okubo, T. Kamikawa, T. Kanimoto, Y. Inagaki, H. Kikuchi, T. Saito, M. Azuma, and M. Takano, J. Phys. Soc. Jpn. 72, 2464 (2003).

[33] B. Wolf, S. Zherlitsyn, B. Lüthi, N. Harrison, U. Löw, V. Pashchenko, M. Lang, G. Margraf, H. W. Lerner, E. Dahlmann, F. Ritter, W. Assmus, and M. Wagner, Phys. Rev. B 69, 092403 (2004).

[34] G. Quirion, T. Taylor, and M. Poirier, Phys. Rev. B 72, 094403 (2005).

[35] B. Wolf, S. Zherlitsyn, S. Schmidt, B. Lüthi, H. Kageyama, and Y. Ueda, Phys. Rev. Lett. 86, 4847 (2001).

[36] A. Sytcheva, O. Chiatti, J. Wosnitza, S. Zherlitsyn, A. A. Zvyagin, R. Coldea, and Z. Tylczynski, J. Low Temp. Phys. 159, 109 (2010).

[37] V. H. Crawford, H. W. Richardson, J. R. Wasson, D. J. Hodgson, and W. E. Hatfield, Inorg. Chem. 15, 2107 (1976).

[38] E. Gati, S. Köhler, D. Guterding, B. Wolf, S. Knöner, S. Ran, S. L. Bud'ko, P. C. Canfield, and M. Lang, Phys. Rev. B 86, 220511(R) (2012).

[39] K. E. Evans, Endeavour, New Series 15, 170 (1991).

[40] Note that the anomalous length changes around $T_{N}$ discussed above, giving rise to a negative Poisson effect at low temperatures, are of the order of $\Delta l / l \leqslant 10^{-5}$ and therefore beyond the resolution of standard structural investigations.

[41] F. Ruszala and E. Kostiner, J. Cryst. Growth 26, 155 (1974). 